\title{
Propuesta de aprendizaje bimodal para mejorar los primeros cursos de matemática en la universidad. La situación de la Universidad Nacional
}

\author{
Proposal of blended learning to improve the \\ first mathematics courses at the University. \\ The situation of the Universidad Nacional
}

Yuri Morales-López' 


\section{Palabras clave}

Matemáticas universitarias; tecnologías de la información y comunicación (TIC); educación matemática; Moodle.

\section{Resumen}

El curso Matemática general, código MAX084, de la Universidad Nacional de Costa Rica está diseñado para fortalecer los conocimientos básicos en matemáticas y así asegurar que los estudiantes dispongan de los requisitos para un curso de cálculo. Pero los resultados obtenidos desde 2007 evidencian debilidades y dificultades de los cursantes. Entre los múltiples factores involucrados están el escaso tiempo con que se cuenta en las sesiones presenciales para ofrecer más ejemplos relevantes, tiempo para reforzar conocimientos de secundaria y cantidad de ejercicios que se pueden revisar. En esta propuesta se crea un insumo para el mejoramiento de la actividad en este curso, impartido por la Escuela de Matemática de la Universidad Nacional de Costa Rica (UNA) a diferentes carreras y facultades. El objetivo es transformar el entorno tradicional de este curso mediante la incorporación de lo virtual en las prácticas de enseñanza utilizando la plataforma Moodle. Esto supone una evolución de los procesos actuales del quehacer académico en la UNA, de las herramientas disponibles y un cambio cultural en todo el proceso.

\section{Keywords}

College mathematics; information and communications technology (ICT); mathematics education; Moodle.

\begin{abstract}
The General Mathematics course code MAX084 is designed to strengthen basic skills in mathematics precollege and thus ensure that students have the requirements for a calculus course. But the results obtained since 2007 reveal certain weaknesses and difficulties of students. Among the many factors involved are the small amount of time in which is told in the sessions to provide more relevant examples, time to strengthen high school knowledge and the amount of exercise to review. This proposal creates an input for the improvement of the activity in the General Mathematics MAX084 course taught by the Escuela de Matemática de la Universidad Nacional de Costa Rica (UNA) to different careers and colleges. The goal is a proposal to transform the traditional setting of MAX084 General Mathematics course by incorporating the virtual in teaching practices with the use of the Moodle platform. This is an evolution of the current processes of existing academic work at UNA, the tools available and a cultural change throughout the process.
\end{abstract}

\section{Introducción}

Las expectativas sobre la educación cambian continuamente, proporcionando un medio útil para redireccionar los esfuerzos. Si se parte de esto, y además de una educación integral y repensada constantemente, muchas dificultades sobre contextualización y sentido de pertenencia sobre el proceso podrían ser atenuadas. Aún así, muchos otros fenómenos internos y externos están presentes. Actualmente, no solo es cuestión de interés si se adquiere conocimiento e información, sino de si es posible utilizarlo y que este uso sea adecuado.

Junto a esto, los cambios en cada etapa del proceso educativo suponen uno de los tantos fenómenos que pueden influir sobre el aprendizaje de los estudiantes. Como el cambio de primaria a secundaria, la inclusión en la educación superior también conlleva cierta dificultad por los ritmos y tareas que cada etapa supone. En la educación media, en términos universales, se espera poder formar un ciudadano con conocimiento y cultura general. Cada país normalmente ha determinado ciertas características de estos egresados.

En la educación superior, los objetivos son distintos; querer definirlos supondría un acercamiento ineludible a la comprensión de lo que es la profesionalización. Pero junto a la delicada necesidad de formar profesionales subyace una tarea aún más 
loable: formar profesionales integrales. En este contexto, no solo se espera un profesional capacitado para realizar una o varias tareas específicas, sino que también supere las expectativas del conocimiento ornamental (como los libros en una repisa) y logre utilizar las herramientas que la educación le ofrece para vivir en armonía con su ser, con los demás y con el planeta.

Otro elemento fundamental es que no se puede seguir percibiendo la matemática y su enseñanza como un acto aislado propio del quehacer de un matemático. Como señala De Guzmán (2007):

En la comunidad matemática internacional se viene prestando recientemente una gran atención a los medios convenientes para lograr abrir los ojos de amplios sectores de la sociedad hacia los beneficios de todos los órdenes que puede reportar una cultura que integre, del modo debido, ciencia y matemática. (párr. 26).

Está claro que esta misión no se alcanza aisladamente de los otros procesos como primaria y secundaria; existen nuevas tareas y objetivos en esta nueva etapa de formación. La educación matemática en la universidad también debe estimular y fortalecer los grandes objetivos ya mencionados. Lamentablemente, al menos en Costa Rica, todavía está arraigado un sentimiento de aversión hacia el aprendizaje de las matemáticas. Muchos son los factores involucrados en esto: una mala formación en etapas anteriores, hábitos de estudio y herencias sobre supuestos culturales, entre muchos otros. Autores como Ruiz (2013) lo interpretan así:

La Matefobia: esta condición de naturaleza cultural, que trasciende sin duda el sistema educativo, conspira para limitar condiciones socioafectivas apropiadas en los estudiantes, padres y madres de familia e incluso docentes a la hora de realizar una construcción de aprendizajes matemáticos significativos y edificantes. (p. II)

En este documento se presenta una propuesta que intenta abordar y ofrecer un insumo para el mejoramiento de la actividad en el curso Matemática general MAX084, impartido por la Escuela de Matemática de la Universidad Nacional (UNA) a diferentes carreras y facultades.

Tal propuesta consiste en un plan para apoyar al estudiantado desde un modelo no tradicional de educación para la UNA. Se emprende la tarea de procurar, desde el modelo de educación a distancia, un apoyo para la acción dentro del aula y fuera de esta, y gestionar una oportunidad para rasgar ciertas barreras normales en la educación presencial como el acceso, tiempo de contacto y otros recursos. En los próximos párrafos se señala el problema específico, una breve descripción de la propuesta pedagógica y los objetivos de este proyecto.

\section{Situación problemática por atender}

La educación en matemáticas es una de las necesidades de todos los profesionales. Sin embargo, no necesariamente ser educado en matemáticas responde a saber únicamente técnicas y algoritmos, saber cálculo, métodos numéricos o álgebra lineal. La matemática universitaria es una poderosa herramienta en cada disciplina y otorga una ventaja para poder crear nuevos conceptos. La matemática universitaria tiene el fin de proporcionar tres grandes elementos para el trabajo y para la vida:

I. Crear una estructura de pensamiento lógico formal

2. Saber resolver problemas

3. Dominar un lenguaje para describir los fenómenos

En esta importante tarea, la estructura universitaria debe ofrecer las mejores circunstancias para asegurarse de que el estudiante adquiera las condiciones básicas para desarrollar una nueva estructura mental. Entre estos contextos primordiales está la imperiosa necesidad de saber si realmente el estudiante podrá afrontar los retos que un curso de matemáticas universitarias representa. Un asunto relevante es que este curso tiene dos aspectos que debe atender: I) la formación matemática de secundaria (ya sea de alta o baja calidad) y 2) temas básicos no contemplados en el programa de estudio de secundaria.

Esto implica que no solo depende de la formación que se ofrece en el curso sino también de las estrategias con las que el estudiante fue preparado; esto aunado, como se mencionó, a que el curso resume en varias semanas los cinco años de preparación secundaria y anexa otros temas. Junto a esto, varias fuentes señalan que la promoción y aprovechamiento del curso no son lo que se espera. Al respecto, se pueden mencionar: 
1. Las notas de aprobación y reprobación del curso (insumo cuantitativo).

2. Los informes finales de los cursos por parte de los coordinadores (insumo cualitativo).

3. La gran cantidad de deserción de los cursos (insumo cuantitativo).

Se puede adelantar que entre los documentos consultados aparecen varias debilidades expresadas por los estudiantes y recogidas por el personal docente coordinador. Entre otras:

I. Falta de estudio.

2. Mala formación en secundaria.

3. Tiempo en que se imparte el curso versus la cantidad del contenido.

Luego de algunos años de ofrecer el curso, se han considerado varias estrategias en la evaluación con el fin fortalecer el apoyo al estudiante: I) Ampliar la cantidad de instrumentos de evaluación como tareas, proyectos, exámenes cortos, exámenes parciales; 2) ampliar las horas de consulta por el docente a cargo y contratación de profesionales en matemática educativa para programas continuos de consulta, entre otros; pero los resultados no han variado.

En síntesis:

El problema que se desea atender con esta propuesta es la falta de alternativas para el fortalecimiento del quehacer del estudiante, considerando la escasa cantidad de tiempo con que se cuenta en las sesiones presenciales para ofrecer ejemplos relevantes, reforzar conocimientos de secundaria y número de ejercicios que se puede revisar.

\section{Marco teórico}

\section{Condiciones del estudiantado}

Regularmente, el estudiante toma este curso en su primer año universitario. Por la normativa nacional, todos los estudiantes deben haber aprobado secundaria para ingresar a las universidades.

Varias debilidades de los programas de secundaria anteriores ya han sido documentadas en Ruiz (20/3).
Para graduarse de secundaria, se debe aprobar el examen nacional llamado Pruebas Nacionales de Bachillerato en las principales áreas (incluida matemáticas); su objetivo es verificar la calidad de la educación brindada. El promedio relativo calculado por el $60 \%$ de la nota obtenido en el examen sumado a la nota de presentación que representa el 40\% determinan la promoción estudiantil. ${ }^{2}$ En general, la promoción de estudiantes en este periodo es muy baja, en el cuadro I se muestran los promedios de aprobación de 2005 a 2009.

En un estudio realizado en 2003, Barrantes (citado por Cordero y Rojas, 20I I) señala que solamente la mitad de los docentes de matemáticas considera que los contenidos desarrollados en bachillerato son apenas suficientes para la formación universitaria.

Debido a lo anterior, se evidencia que la enseñanza en la Educación Media se encuentra dirigida a "ganar el examen", por lo que se genera poca oportunidad para desarrollar estrategias de aprendizaje que resulten significativas para el estudiante (Barrantes, 2003, citado por Cordero y Rojas, 20 I I, p. 9).

Otro insumo para conocer la situación de los estudiantes que ingresan a este curso es la prueba de diagnóstico que aplican las universidades públicas. Aunque existen cuantiosos datos numéricos que podrían servir para evidenciar la escasa formación, basta señalar que, en promedio, ni la mitad de los estudiantes logra aprobar un examen de conocimientos previos. Esto ya supone un fracaso inicial de la mitad de los estudiantes en los respectivos cursos de matemáticas.

\section{Condiciones del curso}

El curso Matemática general MAX084 cuenta con I6 semanas lectivas (más una de exámenes finales) y cinco horas semanales. Está diseñado para fortalecer los conocimientos básicos en matemáticas de colegio y así asegurar que el estudiantado disponga de los requisitos para un curso de cálculo (u otros posteriores).

2 Esta nota se define como el promedio de las calificaciones obtenidas en la educación diversificada en Estudios Sociales y Cívica, Español, Inglés o Francés, Matemáticas y Biología, Química o Física. (Cordero y Rojas, 201 I). 
Cuadro I. Resultado de la Prueba de Bachillerato de Matemática (formal) sin incluir nota de presentación.

\begin{tabular}{|c|c|c|c|c|c|}
\hline Materia & 2005 & 2006 & 2007 & 2008 & 2009 \\
\hline Matemática & 72,77 & 63,63 & 66,67 & 64,64 & 65,53 \\
\hline
\end{tabular}

Fuente: Cordero y Rojas (201 I).

Este se ofrece los dos periodos del año y en promedio consiste de 18 grupos de 40 personas para el primer semestre y 12 grupos de 40 personas para el segundo (en el primer semestre de 2014 se crearon 23 grupos). Las carreras que actualmente lo absorben son: Biología Marina, Biología Tropical, Enseñanza de las Ciencias, Acuacultura, Agronomía, Forestales, Geografía, Cartografía, Diseño, Administración, Relaciones Internacionales y Gestión Ambiental. Se debe señalar que la matrícula no es por carrera sino que todos los estudiantes pueden seleccionar el curso junto a otros de diferentes carreras.

Por otro lado, el código MAX084 aparece en 2007, esencialmente por el cambio de contenidos del curso antecesor, Matemática básica, que se deja de ofrecer ese año (20।3). Los docentes son contratados por 10 horas semanales y su quehacer contempla cinco horas de docencia presencial, dos horas de consulta para los estudiantes y las otras tres horas se distribuyen para planeamiento y reuniones.

Los estudiantes cuentan con un laboratorio dedicado a medio tiempo para el uso de las 40 computadoras en la Escuela de Matemáticas. Además, la UNA dispone de al menos 200 computadoras disponibles para estudiantes en distintos centros y laboratorios.

\section{Metodología}

\section{Objetivo general}

A continuación se señalan los objetivos de la propuesta. Con las condiciones mencionadas anteriormente, se define el objetivo general de este trabajo: Transformar el entorno tradicional del curso de Matemática general MAX084 mediante la incorporación de lo virtual en las prácticas de enseñanza, con el uso de la plataforma Moodle.

\section{Objetivos específicos}

- Desarrollar el curso Matemática general MAX084 en un ambiente educativo a distancia en la plataforma institucional Moodle.
- Desarrollar las unidades didácticas del curso Matemática general MAX084 en un ambiente educativo a distancia en la plataforma institucional Moodle.

- Definir una batería de ejercicios para el curso Matemática general MAX084 para implementar en Moodle.

\section{Planificación operativa}

En el escenario ideal, la primera situación es que la cantidad de personas por grupo supera la capacidad para ofrecer una atención personalizada. En las mejores condiciones, independientemente de ser presencial, se deben atender, a lo sumo, 30 estudiantes.

De esta manera, la necesidad de ofrecer mejores oportunidades para las personas podría ser traducida por la creación y apertura de cursos bimodales o completamente a distancia. Ya muchas carreras en la UNA reconocen la necesidad de ofrecer este tipo de modelo bimodal, aunque no exista el soporte legal de la institución. En este escenario, se podría esperar que docentes y estudiantes posean las mejores condiciones iniciales respecto al uso de recursos tecnológicos, donde al menos se conozcan las dinámicas básicas del uso de internet.

Respecto a la cantidad de recursos, se debe indicar que es necesario brindar más espacios a los estudiantes para que tengan acceso a los laboratorios u otro tipo de facilidades similares. Esto no es trivial; la experiencia indica que muchos estudiantes aun en segundo año de sus carreras no tienen o no saben manejar una cuenta de correo.

Por supuesto, hay otra debilidad que no puede escapar de la proyección: el tiempo de estudio. Este fenómeno de pocas horas invertidas en estudio es complicado, pero indiscutiblemente es necesario que el estudiante también asuma parte predominante de la responsabilidad en su educación.

En el escenario posible, se cuenta con una limitante importante, pues la UNA no permite la evaluación 
con recursos tecnológicos como correos electrónicos, foros, chats y estrategias no presenciales (esto parece un poco contradictorio, pues varias directrices estimulan el uso de la plataforma institucional Moodle). De esta manera, en el escenario posible será difícil llevar a cabo una evaluación conducente directamente a la aprobación del curso; pero el foco del trabajo está orientado al apoyo para el estudiantado. Se podrá ofrecer un espacio para el desarrollo del curso con materiales propiamente diseñados para el trabajo a distancia, una extensa batería de ejercicios para el desarrollo de las nociones elementales sobre algoritmos que son fundamentales en el desarrollo de este curso. Por lo anterior, se espera que en un año la propuesta del curso mencionado esté alojada en el Moodle institucional de la UNA.

Se caracterizará de la siguiente manera: material didáctico propio, mediado pedagógicamente y de acuerdo con lo que posibilita la web 2.0. Se espera que pueda ofrecerse el curso para establecer planes piloto (sobre este mismo curso) y la posibilidad de invertir parte del tiempo de planeación del curso para realizar tareas de seguimiento personalizado. Aunque en un escenario ideal futuro, se espera que tal curso se pueda pilotear con profesores y profesoras, para utilizarlo como recurso en todos los cursos que se imparten en esta cátedra.

Este asunto también enfatiza la necesidad de crear una cultura sobre la actividad de aprendizaje en este tipo de entorno y de la cual se espera, paulatinamente, tener mayores destrezas y competencias en este tipo de aprendizaje (tanto en docentes como en estudiantes). En este sentido, Prieto (20/2) resume este tipo de propuesta con "un manejo todavía centrado en la presencialidad con creciente utilización de tecnologías, una entrada fuerte de éstas para la realización de prácticas fuera del aula o bien una propuesta en que el entorno está constituido por lo virtual" (p. 4). Esto supone una evolución de los procesos actuales del quehacer académico en la UNA, de las herramientas disponibles y un cambio cultural en todo el proceso.

Esta propuesta presume partir de un entorno presencial, donde se incluirán actividades alternativas de trabajo en línea como prácticas y tareas para realizar dentro del sistema de trabajo. Se incorporan estas actividades previas al inicio del curso y se planifican antes de su apertura. Como se mencionó en el apartado anterior, posiblemente tener disposición institucional para el trabajo a distancia o en línea conlleve algún tiempo y experiencias documentadas.

\section{Propuesta pedagógica}

El curso aborda una gran cantidad de contenidos. Esto implica que el compromiso de los estudiantes es mucho mayor que en otros cursos iniciales en la UNA. Junto a esto, se mencionó que el curso presupone algunos conceptos básicos que no necesariamente los estudiantes están adquiriendo en los procesos de primaria y secundaria. Este curso parte del álgebra de secundaria; sin embargo, muchas de las operaciones que admiten los números reales no han sido comprendidas a cabalidad. Basta ver la cantidad de veces que los estudiantes anteponen una suma ante un producto. Esto no es nada extraño, pues se heredan muchos errores aritméticos al trabajo algebraico. Esto admite un abordaje pedagógico distinto al presencial. Junto a reglas y ejemplos iniciales, será necesario un material que guíe al estudiantado y considere las necesidades que en el ambiente presencial no es posible atender.

Muchos de estos conocimientos también pueden ser adquiridos por reforzamiento, pues son las reglas iniciales básicas: sumar, restar, multiplicar y dividir. Así, varias actividades serán relevantes para esta propuesta y su sustento pedagógico: guías didácticas, ejemplos y videos, foros y una muy amplia batería de ejercicios. No se supone la sustitución de la acción que se realiza en el ambiente presencial, pero ciertas conductas son necesarias para que esta propuesta de trabajo sea factible:

I. Invertir en tiempo de estudio por parte del estudiantado.

2. Desarrollar las clases y unidades (los materiales, ejemplos, guías, foros, entre otros).

3. Seguimiento del avance de estudiantes y atención de consultas.

Es claro que el juicio que gira en torno a este tipo de trabajo no solo involucra los factores ya señalados, sino también una concepción de educación. Finalmente, esta propuesta se sostiene sobre una pedagogía conductista con algunas actividades de tipo constructivista. Esto guiará muchas de las rutas que se trabajarán durante el curso, pero también se trabajará sobre un aprendizaje lo mejor contextualizado y significativo para el estudiante. 


\section{Resultados esperados de la propuesta}

En el contexto en que se han señalado las acciones, es importante suscribir este trabajo en un entorno de aprendizaje e-learning, concebido como herramienta de gestión del conocimiento que, según Prieto y Van de Pol (2006), funciona principalmente como un lugar o sitio donde se tiene la información en conjunto y desde ahí se puede tener acceso a otros lugares, sitios y recursos.

Se espera con esto que se logre capacitar a 40 estudiantes en la etapa piloto con un profesor $y$, posteriormente, a los grupos en total. Inicialmente se investigará con un profesor a cargo de la coordinación y ejecución del curso y, luego, se extenderá a cinco docentes de matemática.

En términos generales se espera disponer de:

I. Cinco profesores capacitados para ofrecer este curso.

2. Una prueba piloto, desarrollada, probada y en marcha.
3. Una metodología de enseñanza y aprendizaje de e-Learning como herramienta de gestión de conocimiento.

4. Tres unidades de trabajo en línea.

\section{Mapa de prácticas de aprendizaje}

En este tipo de propuesta se hace necesario conocer cuáles actividades se desarrollarán para prever, entre otras cosas, los recursos tecnológicos disponibles. A continuación se detallan las actividades que se proponen en el curso:

Es preciso recordar que el tutor o tutora será la persona docente a cargo del curso, por lo que esta matriz no podrá ser tan amplia como se desearía en última instancia. El objetivo es que al definir actividades claras se pueda planificar y crear los materiales para trabajar en estas.

\section{Tecnologías apropiadas}

Para la ejecución de esta propuesta se debe contar, respecto a hardware, con una computadora con

\begin{tabular}{|c|c|c|}
\hline Semana & Actividad* & Descripción* \\
\hline । & $\begin{array}{l}\text { Bitácora } \\
\text { electrónica }\end{array}$ & $\begin{array}{l}\text { Durante el curso se le pide al estudiantado que, al menos una vez por semana, autoevalúe su } \\
\text { proceso de aprendizaje en un blog (por ejemplo, qué aprendieron esa semana, qué les resultó } \\
\text { de especial relevancia, etc.). A través de todo el curso. }\end{array}$ \\
\hline \multirow{3}{*}{2} & Lectura & $\begin{array}{l}\text { Como ejercicio o tarea, se le da al estudiantado una lista de enlaces a artículos sobre temas } \\
\text { determinados, entre los cuales pueden escoger uno. }\end{array}$ \\
\hline & Foro de debate & $\begin{array}{l}\text { Después de tratar un tema controvertido, el docente abre un nuevo hilo en el foro de } \\
\text { discusión. Se le pide al estudiantado que describa su reacción inicial al tema. El docente dirige } \\
\text { la discusión con cuidado. }\end{array}$ \\
\hline & Lectura & $\begin{array}{l}\text { Como ejercicio o tarea se le da al estudiantado una lista de enlaces a artículos sobre temas } \\
\text { determinados, entre los cuales pueden escoger uno. }\end{array}$ \\
\hline \multirow[b]{2}{*}{5} & Ensayo & Cada estudiante escribe un ensayo y lo sube a la página del curso. \\
\hline & Examen corto & $\begin{array}{l}\text { Al final del módulo se le pide al estudiantado que responda a una serie de preguntas multiple- } \\
\text { choice (preguntas de opción múltiple o examen tipo test). El resultado será inmediatamente } \\
\text { visible para el estudiantado. }\end{array}$ \\
\hline 12 & Examen corto & Ídem \\
\hline 15 & Presentación & $\begin{array}{l}\text { Cada estudiante, de manera individual, compila su tema de investigación, su investigación o } \\
\text { exploración y la sube a la web del curso. Les pide a sus compañeros y compañeras que hagan } \\
\text { una devolución de los descubrimientos y la presentación. }\end{array}$ \\
\hline 16 & Presentación & $\begin{array}{l}\text { Cada estudiante, de manera individual, compila su tema de investigación, su investigación o } \\
\text { exploración y la sube a la web del curso. Les pide a sus compañeros y compañeras que hagan } \\
\text { una devolución de los descubrimientos y la presentación. }\end{array}$ \\
\hline
\end{tabular}


acceso a internet, disponible a tiempo completo, para el personal docente a cargo y con el acceso a los laboratorios para el estudiantado. En cuanto al uso de software, se requerirá crear un curso en Moodle en concordancia con la propuesta y asignar el perfil de gestor. Es importante indicar que los laboratorios, en su mayoría, tienen computadoras para ejecutar applets y rutinas java que serán necesarias en el proceso. Además, será preciso el proceso de matrícula de los estudiantes.

\section{Materiales didácticos, tutorías y sistema}

Se desarrollarán tres unidades de trabajo para el tratamiento de los temas propuestos en la sección Mapa de prácticas. Estas unidades deberán estar desarrolladas antes de la ejecución del curso. En el caso de las tutorías, se desarrollarán por parte del docente a cargo y se atenderá por medio de foros y correo interno. Se debe coordinar la presentación de reporte de horas tanto presenciales como en la plataforma institucional, según la cantidad de atención al estudiantado. En este sentido, se pretende destinar una hora semanal distribuida por la carga académica en la cual se tomarán las responsabilidades de tutoría en el espacio virtual. Se procura que cada docente que asuma la estrategia de trabajo complete un proceso de capacitación para que las actividades sean pertinentes.

En el caso de la administración de la plataforma, existen dos personas asignadas a la plataforma institucional, que manejan los perfiles de administradores y otorgan la capacidad para gestionar los cursos. Además, el equipo del Programa UNA-Virtual se encarga del mantenimiento y actualización de toda la plataforma.

\section{Indicadores de evaluación}

En este apartado se abordan las principales nociones sobre la coherencia del proyecto, su seguimiento y evaluación. El propósito es preservar la coherencia entre los objetivos, las metas planteada y las acciones que evidenciarán los avances y alcances. Como se indicó en el apartado anterior, este plan proyecta su operación en el modelo pedagógico general, en las prácticas de aprendizaje y tecnologías, en el material didáctico, en la tutoría y en la administración.

\section{Indicadores}

Respecto a las evidencias sobre el desarrollo de la propuesta, a continuación se detallan los indicadores que serán considerados. Se utiliza la estrategia desarrollada por Becerra y otros (2004).

\section{Modelo pedagógico general}

I. Es deseable que exista acompañamiento del departamento de diseño curricular de la UNA para valorar las acciones en el currículo actual.

II. Es indispensable que exista apoyo de la Dirección de la Escuela de Matemáticas para el desarrollo de una estrategia curricular distinta.

III. Es deseable que exista compromiso del estudiantado, personal docente y administrativo de la plataforma y del área administrativa de la Escuela de Matemática.

\section{Prácticas de aprendizaje y tecnologías}

I. Es deseable la participación estudiantil en las prácticas sugeridas.

II. Es indispensable que se cuente con una computadora con acceso a internet para el diseño y ejecución del curso.

\section{Material didáctico}

I. Es necesario que se desarrollen los materiales educativos en correspondencia con las nuevas acciones planteadas en esta propuesta.

II. Es deseable que los docentes identifiquen y desarrollen propuestas para la actualización y gestión de los materiales.

Tutoría

I. Es deseable crear espacios en las sesiones presenciales de tutoría para ofrecer seguimiento de los ejercicios y prácticas. Esto, considerando que el proceso será evolutivo e inicialmente el estudiantado necesitará aclarar algunas dudas.

II. Es necesario contar con un estudiante asistente que colabore con la digitación de ejercicios.

\section{Administración}

I. Es deseable contar con espacios específicos en el laboratorio de la Escuela de Matemática de la UNA para garantizar un lugar donde el estudiantado pueda realizar sus actividades. 
II. Es indispensable la comunicación con el Departamento del Programa UNA Virtual que administra la plataforma educativa.

\section{Algunas consideraciones}

Como ha sido desarrollado a través de este documento, la planificación de ambientes de aula que favorezcan la incorporación de recursos tecnológicos no aparecerá espontáneamente con la implementación del recurso hardware. Más allá de esto, las rutas para este tipo de ambientes requieren una reorganización del currículo del curso y de los otros materiales disponibles.

Es fácil encontrar muchas experiencias que se reducen a la implementación de una o dos sesiones en donde se utiliza Geogebra® u otros recursos para introducir un tema o guiar una exposición donde se reduce todo el entorno, comúnmente tradicional, a un espacio de algunos minutos para este fin.

La bibliografía internacional ya muestra que la investigación en educación matemática y TIC ha avanzado lo suficiente para sobrepasar estas pseudoactividades y proponer un entorno distinto de aprendizaje (incluso entornos bimodales o completamente virtuales). Los aportes de estas actividades posiblemente cautiven al profesorado novato en la incorporación de tecnologías, tal como ocurrió hace varios años con las diapositivas y el videobeam.

Esta propuesta supone un cambio paulatino hacia la creación de recursos de forma inteligente: abarca no solo espacios de incorporación sino que establece los elementos primordiales para que el impacto del uso de los recursos en el aula de matemática sea provechoso. Morales y Poveda (2013, p. 7) indican que:

Es evidente que la suma de pequeños esfuerzos no es suficiente para entablar y encarar una problemática que posee múltiples aristas (económicas, sociales, tecnológicas, entre otras). Usar un software, un retroproyector, acceso a Internet y el email, de forma desligada, ofrecen muy pocos elementos claros para desafiar las rutas tradicionales de aprendizaje (p. 7).

Instrumentos de seguimiento que se utilizarán en la etapa de pilotaje y ejecución

\begin{tabular}{|c|c|c|}
\hline Fase & Instrumento & Relación con el mapa de prácticas de aprendizaje \\
\hline \multirow{4}{*}{ 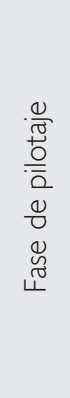 } & $\begin{array}{l}\text { Encuesta (cerrada) sobre percepción inicial } \\
\text { del curso. }\end{array}$ & $\begin{array}{l}\text { Es la primera actividad para reconocer algunas de las condiciones } \\
\text { particulares. }\end{array}$ \\
\hline & $\begin{array}{l}\text { Entrevista (semidirigida) sobre percepción } \\
\text { de la ejecución del curso. }\end{array}$ & $\begin{array}{l}\text { Esto concuerda con la entrega de la revisión de las actividades lectura y } \\
\text { ensayo de la semana } 5 \text { en el mapa de prácticas de aprendizaje. }\end{array}$ \\
\hline & Bitácora & Concuerda con el cierre del curso. \\
\hline & $\begin{array}{l}\text { Encuesta (cerrada) sobre percepción final } \\
\text { del curso. }\end{array}$ & Concuerda con el cierre del curso. \\
\hline \multirow{5}{*}{ 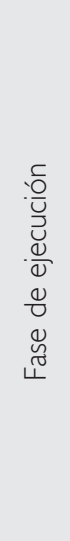 } & $\begin{array}{l}\text { Encuesta (cerrada) sobre percepción inicial } \\
\text { del curso. }\end{array}$ & $\begin{array}{l}\text { Es la primera actividad para reconocer algunas de las condiciones } \\
\text { particulares. }\end{array}$ \\
\hline & $\begin{array}{l}\text { Encuesta (cerrada) sobre percepción inicial } \\
\text { del curso. }\end{array}$ & $\begin{array}{l}\text { Es la primera actividad para reconocer algunas de las condiciones } \\
\text { particulares. }\end{array}$ \\
\hline & $\begin{array}{l}\text { Entrevista (semidirigida) sobre percepción } \\
\text { de la ejecución del curso. }\end{array}$ & $\begin{array}{l}\text { Esto concuerda con la entrega de la revisión de las actividades lectura y } \\
\text { ensayo de la semana } 5 \text { en el mapa de prácticas de aprendizaje. }\end{array}$ \\
\hline & $\begin{array}{l}\text { Encuesta (cerrada) sobre percepción final } \\
\text { del curso. }\end{array}$ & Concuerda con el cierre del curso. \\
\hline & $\begin{array}{l}\text { Encuesta (cerrada) sobre percepción final } \\
\text { del curso. }\end{array}$ & Concuerda con el cierre del curso. \\
\hline
\end{tabular}


Una restructuración del modelo pedagógico general, las prácticas de aprendizaje y tecnologías, el material didáctico, las tutorías y la administración de los recursos es apenas el inicio en el camino por comprender el verdadero papel de los recursos tecnológicos en el quehacer del educador y la educadora en matemáticas.

En el caso de la UNA, específicamente la Escuela de Matemática, tendrá que redireccionar ciertos elementos en su oferta de servicio. Se está viviendo un cambio transcendental en la formación preuniversitaria con los programas de matemáticas aprobados en 2012 y que se comenzaron a implementar en 2013.

Aunque es pronto para que las universidades vean los resultados de estos programas educativos, tienen la tarea ineludible de atender a los futuros profesionales formados con los antiguos programas y trascender su actividad actual para crear una estructura para los cursos de otras universidades que estén a la altura de los programas actuales.

Si no se impone desde ya la necesidad de reformular la actividad del estudiantado universitario en los cursos de matemáticas iniciales, es posible que en pocos años las universidades enfrenten una importante desconexión entre la formación preuniversitaria y la impartida en la universidad.

Propuestas como la aquí consignada buscan crear un nuevo marco de formación de profesionales en áreas medulares de la sociedad costarricense. Para esto, se deberán aprovechar los recursos tecnológicos y todas las herramientas disponibles, con el fin de aportar espacios de construcción de conocimiento donde se tengan recursos alternos a la presencialidad impuesta por la vida universitaria tradicional, especialmente en la UNA.
Esta propuesta específica involucra al personal docente, estudiantado, equipo, infraestructura, atención técnica, edición de materiales impresos y digitales, actividades y prácticas, entre otros. Pero más allá, busca crear sinergia entre actores y recursos para afrontar los retos que el nuevo contexto educativo costarricense impone.

\section{Bibliografía}

Becerra, M. et al. (2004). Informe Final. Comisión Asesora de Educación a Distancia. Ministerio De Educación, Ciencia y Tecnología. Documento en línea. Obtenido desde: http:// www.coneau.gov.ar/archivos/477.pdf.

Cordero, M. \& Rojas, M. (20 I I). El conocimiento matemático previo del estudiante y su relación con el desempeño académico en el curso MAX084 Matemática general de la Universidad Nacional. (Tesis de licenciatura). Universidad Nacional.

De Guzmán, M. (2007). Tendencias innovadoras en educación matemática. Obtenido desde: http://www.mat.ucm.es/catedramdeguzman/drupal/migueldeguzman/legado/educacion/ tendenciaslnnovadoras.

Morales, Y. \& Poveda, R. (20|3). Plataforma educativa nacional para la formación continua de docentes de matemáticas en Costa Rica. En: Rodríguez, E. Actas del VII Congreso Iberoamericano de Educación Matemática CIBEM 7, 70307037. Montevideo. Obtenido desde: http://www.cibem. org/7/actas/pdfs/53.pdf.

Prieto, D. (2012). Fase de planificación operativa. Planificación, seguimiento y evaluación de proyectos. Instituto de Formación Docente de Virtual Educa.

Prieto, D. \& Van de Pol, P. (2006). E-Learning, comunicación y educación. El diálogo continúa en el ciberespacio. Bogotá.

Ruiz, A. (20 I 3). La reforma de la educación matemática en Costa Rica. Perspectiva de la praxis. Cuadernos de Investigación y Formación en Educación Matemática 8 (especial). Obtenido desde: http://revistas.ucr.ac.cr/index.php/cifem/article/download/ | | | 25/10602.

Van de Pol, P. (20 I2). Una tipología de las prácticas de e-Learning. Instituto de Formación Docente de Virtual Educa. 